\title{
Esthetic Region Space Closure Using Crestal and Buccal Corticotomy: A Case Report
}

\author{
Mohammad N Alam ${ }^{1}$, Wael Ibraheem², Syed AA Bukhari ${ }^{3}$
}

\begin{abstract}
Aim and objective: Esthetic region space closure using crestal and buccal corticotomy applying the concept of periodontally accelerated osteogenic orthodontics (PAOO).

Background:The concept of corticotomy-mediated orthodontics helps in rapidly moving teeth with minimal effects on the periodontium and reduces the treatment time. The approach toward treating anterior space closure using buccal corticotomy has been in practice, in the present case report we have included the same fundamental concepts of corticotomy and additionally introduced a crestal corticotomy. Periodontally accelerated osteogenic orthodontics is a surgical technique to create corticotomy which helps to reduce the density of the bone and increases the turnover of tissues adjacent to the surgical site, this decrease in the bone density and increase in the bone turnover helps to facilitate the orthodontic tooth movement.

Case description: A patient aged 24 years/male undergoing orthodontic treatment for closure of open spaces in the anterior region, reported with open space between the lateral incisor and canine in the maxillary region, although the treating orthodontist attempted closure using various techniques by sliding mechanism using active tie back and NiTi closed coil spring for 6 months and when the space did not close further attempts were made using non-sliding mechanics with the help of omega loops, the results were negative. The patient was referred to the periodontics department wherein the case was evaluated and surgical correction using PAOO techniques was planned, following the treatment plan the procedure was completed successfully without any complication, and subsequently, 1-2-week follow-up was done the surgical site healing was satisfactory and the sutures were removed, following which the patient was referred to the orthodontist for activation of space closure by NiTi closed coil spring $9 \mathrm{~mm}$ delivering the light force of $150 \mathrm{~g}$.

Conclusion: The results of the procedure were satisfactory healing of the surgical site and there was no complication like pocket formation, recession, or loss of interdental papilla and on activation of NiTi closed coil spring the space closure was achieved.

Clinical significance: The intervention of periodontal surgery by crestal and buccal corticotomy helps to achieve space closure within a short duration with minimal complication. The decrease in bone density and increase in bone turnover helps to facilitate the orthodontic tooth movement to achieve the desired results.
\end{abstract}

Keywords: Alveolar bone, Corticotomy, Osteotomy.

World Journal of Dentistry (2021): 10.5005/jp-journals-10015-1871

\section{BACKGROUND}

Orthodontic therapy, especially adult orthodontics nowadays, is very demanding. Although teeth movements in adult patients are difficult but not impossible. Also, there are very slow movements of teeth in adult patients.

The clinical procedure that combines selective alveolar corticotomy, use of particulate bone grafting, and application of orthodontic forces is termed periodontal accelerated osteogenic orthodontics (PAOO). ${ }^{1}$ The concept of this combined procedure is based on the bone healing pattern which is termed regional acceleratory phenomenon (RAP). ${ }^{2}$ The decrease in bone density and increase in bone turnover helps to facilitate orthodontic tooth movement. The various outcomes of the procedure are:

- Enhances alveolar bone width. ${ }^{3}$

- Reduced treatment time. ${ }^{4}$

- Enhances post-treatment stability. ${ }^{5}$

- Reduced apical root resorption. ${ }^{6}$

Clinical conditions of space closure are sometimes difficult to manage orthodontically especially when relatively more distance between the teeth are present and abnormal bony patterns which act as resistance to space closure. In such situations, orthodontic correction in collaboration with periodontal surgical procedures may be beneficial in the early closure of space. The periodontal
1,2Department of Preventive Dental Sciences, College of Dentistry, Jazan University, Jazan, Kingdom of Saudi Arabia

${ }^{3}$ Dental Department, Armed Forces Hospital, Jazan, Kingdom of Saudi Arabia

Corresponding Author: Mohammad N Alam, Department of Preventive Dental Sciences, College of Dentistry, Jazan University, Jazan, Kingdom of Saudi Arabia, Phone: +966596347760, e-mail: dr. naz.ish.alam@gmail.com

How to cite this article: Alam MN, Ibraheem W, Bukhari SAA. Esthetic Region Space Closure Using Crestal and Buccal Corticotomy: A Case Report. World J Dent 2021;12(6):496-499.

Source of support: Nil

Conflict of interest: None

surgical procedure by altering the alveolar bone architecture may change the resistance of the bony tissue to the orthodontic forces and may favor rapid teeth movement leading to space closure.

A corticotomy is defined as a surgical procedure where only the cortical bone is cut, perforated, or mechanically altered. The medullary bone present is not altered. This is an osteotomy, wherein there is a surgical cut through both the cortical and medullary bone. $^{7}$ 

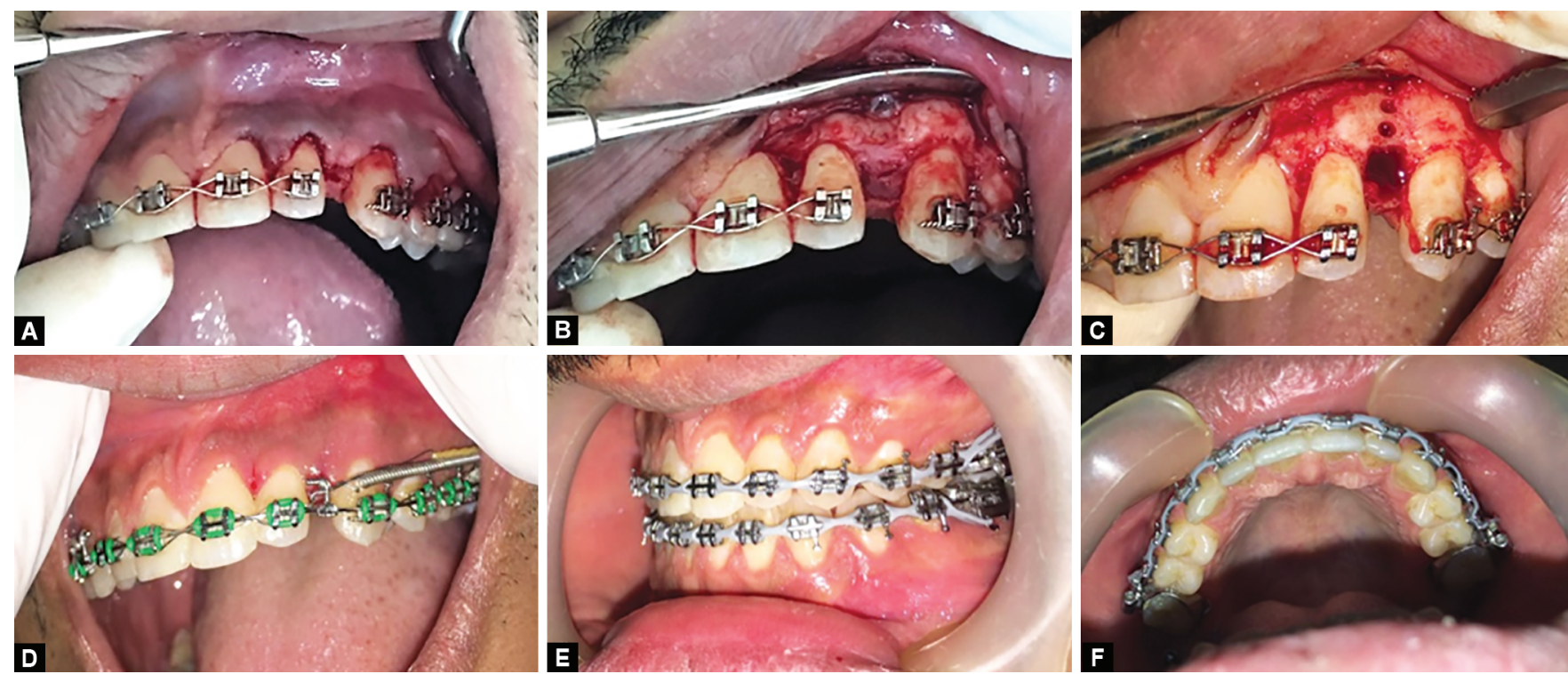

Figs $1 \mathrm{~A}$ to F: (A) Sulcular incision from distal to \#21 to mesial of \#24; (B) Full-thickness flap reflection and exposure of alveolar bone; (C) Crestal osteotomy with buccal corticotomy between \#22 and \#23; (D) Postoperative 1 week placement of closed coil spring for activation of movement; (E) Complete space closure (Buccal aspect); (F) Complete space closure (Occlusal aspect)

The RAP helps in the reduction of bone density and enhances the bone turnover rate, the outcome of this combination procedure facilitates orthodontic tooth movement. The process of RAP initiates within a couple of days from an initial injury, it can have maximum effect at 1-2 months can be prolonged to 4 months, and subside by $6-24$ months. ${ }^{1}$ One important aspect is it can be prolonged to achieve orthodontic tooth movement, hence increasing the predictability and outcome of the treatment. The final re-mineralization of the alveolar bone takes place after the desired orthodontic movements have been achieved to a stable post-treatment outcome.

The surgical procedure for treating anterior space closure using buccal corticotomy has been in practice, in the present case report we have included the same fundamental concepts of corticotomy and additionally introduced a crestal corticotomy. Periodontally accelerated osteogenic orthodontics (PAOO) is a surgical technique to create corticotomy which helps to reduce the density of the bone and increases the turnover of tissues adjacent to the surgical site, this decrease in the bone density and increase in the bone turnover helps to facilitate the orthodontic tooth movement.

\section{Case Description}

A 24-year-old/male patient reported to the College of Dentistry at Jazan University, Saudi Arabia. The chief complaint of spacing in the upper and lower front tooth region. The patient was referred to orthodontics for management of spacing, an orthodontic treatment was planned with non-extraction orthodontic therapy using MBT $0.022^{\prime \prime}$ bracket slot using sliding mechanics for segmental space closure followed by upper and lower fixed retainers for retention.

Clinically at the site of teeth number \#22 and \#23, there was the presence of space following an extensive 2 years of orthodontics treatment. The patient was referred to a periodontist for evaluation and management of the condition, after an overview discussion on the treatment plan rendered to the patient with the treating orthodontist it was understood that the condition of open space was present for almost 2 years, the treating orthodontist attempts to achieve closure of space by sliding mechanism using active tie back and NiTi closed coil spring for 6 months and when the space did not close further attempts were made using non-sliding mechanics with the help of omega loops, but the result was negative.

The clinical examination was done, gingival tissue was healthy, probing depth within normal limits and on palpation, with the finger, the crestal bone was found to be thick and irregular.

A comprehensive treatment plan was devised. The site was accessed by the surgical full-thickness flap, followed by crestal and buccal osteotomy with interradicular corticotomy to achieve PAOO. After the surgery, the patient underwent orthodontic space closure by placing the NiTi closed coil spring $9 \mathrm{~mm}$ delivering a light force of $150 \mathrm{~g}$. Before surgery, the patient was informed about the procedure and related complications accepting which the patient consent was obtained. Subsequently, the surgery was done as planned.

\section{Surgical Procedure}

Site disinfection using $2 \%$ chlorhexidine $(\mathrm{CHX})$ was done at the site of surgery. Under LA 1:100,000 (epinephrine) the site was anesthetized using infiltration technique.

Using a BP blade no. 15 a sulcular incision was made involving the distal surface of \#21 to mesial surface of \#24 (Fig. 1A). A fullthickness flap was elevated buccally and palatally, the flap design was an envelope flap exposing the crestal bone and full length of the tooth surface on the buccal aspect extending to the level of mucogingival junction (Fig. 1B). Using a surgical handpiece and surgical round bur, an equally spaced selective linear interradicular corticotomy was performed on the buccal aspect. On the crestal surface, a rectangular osteotomy was performed to reduce the thick irregular dense crestal bone and was termed as crestal osteotomy (Fig. 1C). The surgery was performed under copious saline irrigation to prevent an increase in temperature of the bone surface during osteotomy. The site was approximated using a silk suture (5-0). Complete closure of flap was attained and no bleeding was detected at the time of discharge. Postoperative instruction and medication were advised, the use of $0.12 \% \mathrm{CHX}$ mouth wash was advised to the patient after 24 hours of the surgery. 
The patient was followed up postoperative 1-2 weeks and the healing was satisfactory and the patient did not complain of any postoperative complication. Following suture removal, the patient was taken up by the treating orthodontist and NiTi closed coil spring $9 \mathrm{~mm}$ delivering the light force of $150 \mathrm{mg}$ was initiated for the final closure (Fig. 1D). Three to four months clinical follow-up showed complete closure of the space (Figs $1 \mathrm{E}$ and F) and complete healing of the soft tissue without any complications associated with periodontal pocket formation, loss of interdental papilla, or recession.

\section{Discussion}

The use of PAOO in the treatment of a patient's occlusal and esthetic correction is very significant and the types of results achieved are a positive step ahead. Based on the various research literatures, the technique has shown various advantages such as enhanced alveolar bone thickness, reduction of treatment time, and increased post-treatment orthodontic stability. ${ }^{3-6}$

It is important to consider the following regarding the prescription of analgesics. The pioneers of the procedure caution to not prescribe analgesics like ibuprofen postoperatively as they are non-steroidal anti-inflammatory drugs (NSAIDs) and studies suggest that NSAIDs can interfere with the production of prostaglandin which can retard the bone growth process, which is critical to PAOO. ${ }^{8}$ Non-steroidal anti-inflammatory drugs given during the first 24 hours following trauma cause inhibition of clotting. Therefore, one should not prescribe NSAIDs before or after undergoing PAOO surgery. ${ }^{9}$

The disadvantage of the procedure is extra cost and morbidity associated with surgical procedure, also most class III malocclusion cannot be treated with this procedure. ${ }^{10}$ It is important to consider that for patients with active periodontal disease, the recession should be excluded from PAOO. Understanding the case selection for PAOO is the key to the outcome of the treatment. For example, a case of bimaxillary protrusion with a gummy smile would benefit from segmental osteotomy than the PAOO. The risk of pain, swelling, and infection following the procedure is always there. ${ }^{11}$ The literatures suggest that the postsurgical patient pain is less when compared with the activation of the archwire. Studies based on patient feedback on pain reported discomfort was more with archwire activation than with the surgical procedure. ${ }^{7}$

Some case reports suggest surgical complications appear to be less as with PAOO. It also suggests that controlled multicenter data are not available currently and objective assessment is not possible. The incidence of root resorption related to PAOO is decreased when compared with conventional treatment, and the occurrence of other possible complications, such as ankylosis and devitalization has not been reported. ${ }^{7}$

One study by Bhattacharya et al. compared the treatment time for the closure of extraction space between conventional orthodontic tooth movement and corticotomy assisted and evaluated the alveolar bone thickness before and after corticotomy procedure using CT scan. They concluded that alveolar corticotomies not only accelerated the orthodontic treatment but also provided the advantage of increased alveolar width to support the teeth as evaluated based on the CT scan analysis. ${ }^{12}$

It is important to note that surgical procedure was done using surgical rotary handpiece using burs and under copious irrigation, but sometimes if the basic protocol of the surgery is not followed the procedure might complicate like necrosis due to overheating of the bone due to the use of rotary high speed surgical burs which lead to delayed healing of the site, also may lead to loss of bone architecture leading to an unpleasant esthetic outcome. To overcome this, recent advances suggest the beneficial use of piezo-surgical units may play a significant role in the outcome of the procedure.

A recent study by Ma et al. where an inpatient having bone fenestration and dehiscence on the buccal aspect of mandibular anterior teeth was treated using pouch design and tension-free closure for PAOO. The site was evaluated using $\mathrm{CBCT}$. The results of the study showed advantages in terms of hard and soft tissue augmentation which showed correction of horizontal and vertical defects in the facial aspect of the mandibular anterior tooth region. $^{13}$

Another recent study by Dukka et al. reported the option for orthodontic movement of periodontally compromised teeth where the combination of PAOO with plasma-rich fibrin enhances osteoinductive effects and improves the healing outcome of both soft and hard tissue. The study had a 3-year follow-up with improved soft tissue and width of keratinized gingiva, also they reported CBCT images which shows no bone loss in the buccal bone. ${ }^{14}$

A genetic study by Zhang et al. using a rat study model with four groups was to evaluate the role of microRNA (miR)-21 during PAOO. The study demonstrated that miR-21 plays a significant role during PAOO-mediated orthodontic tooth movement. ${ }^{15}$

\section{Conclusion}

With the increasing number of a patient wanting orthodontic treatment the patient expectations are to complete the treatment within a short duration, PAOO can be a boon, the surgery helps to decrease the bone density and increase the bone turnover which helps to facilitate the orthodontic tooth movement. Although beneficial outcomes are reported from the treatment, we must consider the case selection and related complications.

\section{References}

1. Wilcko WM, Wilcko T, Bouquot JE, et al. Rapid orthodontics with alveolar reshaping: two case reports of decrowding. Int J Periodont Restorative Dent 2001;21(1):9-19.

2. Pham-Nguyen K. Micro-CT analysis of osteopenia following selective alveolar decortication and tooth movement [Master's Thesis]. Boston, MA: Boston University; 2006.

3. Twaddle BA, Ferguson DJ, Wilcko WM, et al. Dento-alveolar bone density changes following corticotomy-facilitated orthodontics. J Dent Res 2002;80:301.

4. Hajji SS, Ferguson DJ, Miley DD, et al. The influence of the accelerated osteogenic response on mandibular decrowding. J Dent Res 2001;30:180.

5. Nazarov AD, Ferguson DJ, Wilcko WM, et al. Improved orthodontic retention following corticotomy using $\mathrm{ABO}$ Objective grading system. J Dent Res 2004;83:2644.

6. Machado IM, Ferguson DJ, Wilcko WM, et al. Root resorption following orthodontics with and without alveolar corticotomy. J Dent Res 2002;80:301.

7. Murphy KG, Wilcko MT, Wilcko WM, et al. Periodontal accelerated osteogenic orthodontics: a description of the surgical technique. J Oral Maxillofac Surg 2009;67(10):2160-2166. DOI: 10.1016/j. joms.2009.04.124.

8. Nazzal AM, Trojan TM, Green M. Uprighting and periodontally accelerated osteogenic orthodontics as an alternative to surgical crown lengthening. J Clin Orthod 2016;50(8):507-511. 
9. Chackartchi T, Barkana I, Klinger A. Alveolar bone morphology following periodontally accelerated osteogenic orthodontics: a clinical and radiographic analysis. Int J Periodont Restorat Dent 2017;37(2):203-208. DOI: 10.11607/prd.2723.

10. Soltani L, Loomer PM, Chaar EE. A novel approach in periodontally accelerated osteogenic orthodontics (PAOO): a case report. Clin Adv Periodon 2019;9(3):110-114. DOI: 10.1002/cap.10045.

11. Campbell JH. Periodontally accelerated osteogenic orthodontics. J Oral Maxillofac Surg 2017;75(1):6. DOI: 10.1016/j.joms.2016.10. 015.

12. Bhattacharya P, Bhattacharya $H$, Anjum A, et al. Assessment of corticotomy facilitated tooth movement and changes in alveolar bone thickness - a CT scan study. J Clin Diagn Res 2014;8(10):ZC26ZC30. DOI: 10.7860/JCDR/2014/9448.4954.

13. Ma Z, Zheng J, Yang C, et al. A new modified bone grafting technique for periodontally accelerated osteogenic orthodontics. Medicine (Baltimore) 2018;97(37):e12047. DOI: 10.1097/MD.0000000000012047.

14. Dukka H, Gossweiler M, Kishimoto T, et al. Periodontally accelerated osteogenic orthodontics (PAOO) using platelet-rich fibrin alone: a modified approach with a 3-year follow-up. Clin Adv Periodon 2018;8(4):177-181. DOI: 10.1002/cap.10038.

15. Zhang $Y$, Tian $Y$, Yang $X$, et al. MicroRNA-21 serves an important role during PAOO-facilitated orthodontic tooth movement. Mol Med Rep 2020;22(1):474-482. DOI: 10.3892/mmr.2020.11107. 